\title{
National Early Warning Scores (NEWS2) and component physiology in COVID-19 positive hospitalised patients in the West of England
}

\section{Lauren J Scott ( $\sim$ Lauren.Scott@bristol.ac.uk )}

National Institute for Health Research Applied Research Collaboration West; Population health Sciences,

Bristol Medical School, University of Bristol https://orcid.org/0000-0003-3129-5123

\section{Alison Tavaré}

West of England Academic Health Science Network

\section{Elizabeth M Hill}

National Institute for Health Research Applied Research Collaboration West; Population health Sciences,

Bristol Medical School, University of Bristol

\section{Lesley Jordan}

Royal United Hospitals Bath NHS Foundation Trust

\section{Mark Juniper}

West of England Academic Health Science Network; Great Western Hospitals NHS Foundation Trust

\section{Seema Srivastava}

North Bristol NHS Trust

\section{Emma Redfern}

University Hospital Bristol and Weston NHS Foundation Trust

\section{Hannah Little}

West of England Academic Health Science Network; Great Western Hospitals NHS Foundation Trust Anne Pullyblank

West of England Academic Health Science Network; North Bristol NHS Trust

\section{Research Article}

Keywords: National Early Warning Score, COVID-19, Emergency Medicine, Secondary Care, Clinical Assessment

Posted Date: November 20th, 2020

DOI: https://doi.org/10.21203/rs.3.rs-111849/v1

License: (c) (i) This work is licensed under a Creative Commons Attribution 4.0 International License. Read Full License 
Version of Record: A version of this preprint was published at Emergency Medicine Journal on November 20th, 2020. See the published version at https://doi.org/10.1136/emermed-2020-210624. 


\section{Abstract}

Background: COVID-19 is an infectious disease, typically characterised by a dry cough, shortness of breath, fever, fatigue, and/or loss of taste and smell. National Early Warning Scores (NEWS2) are used to detect deterioration in hospital and out-of-hospital settings. The Royal College of Physicians (RCP) recommend NEWS2 for management of COVID-19 patients, but there is little evidence to support this.

Aim: To investigate the relationship between NEWS2 values (and component scores) and hospital mortality in hospitalised COVID-19 patients in the West-of-England.

Methods: Electronically recorded, routinely collected, observational physiological measurements (e.g. pulse, temperature, etc) were collated on all adult patients in participating hospitals between March and June 2020. First and maximum NEWS2 values, and their component scores, were calculated from the physiological measurements. Mortality status was presented by first and maximum NEWS2 and component scores, and areas under the curve (AUC) for 2, 7 and 30-day mortality were calculated.

Results: Of 1,263 patients, $26 \%$ died during their hospital admission, $7 \%$ were admitted to ICU and subsequently discharged from hospital, and $67 \%$ were discharged without ICU.

Most patients had low first NEWS2 values: $50 \%$ NEWS2 $=0-2,27 \%$ NEWS2 $=3-4,14 \%$ NEWS2 $=5-6$, and $9 \%$ NEWS2 $=7+$. However, many patients had a high maximum score: $14 \%$ NEWS2 $=0-2,22 \%$ NEWS2 $=3-4,17 \%$ NEWS2 $=5-6$, and $47 \%$ NEWS2 $=7+$. Patients with higher first and maximum scores were more likely to die, require ICU admission, and have longer length of stay. The AUCs for 2-day, 7-day and 30-day mortality were $0.77,0.70$ and 0.65 , respectively. An increase in most first and maximum component scores were associated with an increased risk of mortality.

Conclusion: NEWS2 is a reasonably good predictor of short-term mortality in this population, and the respiratory components are of the most value individually. These findings support the RCP's recommendations to use NEWS2, alongside clinical judgement, in the assessment of COVID-19 patients.

\section{Key Messages}

What is already known on this subject

- COIVD-19 was declared a global pandemic by the World Health Organisation in March 2020.

- National Early Warning Scores are used to detect deterioration and improve outcomes in the general population.

- Patient physiology may not behave as anticipated in COVID-19 patients.

What this study adds

- COVID-19 patients with higher National Early Warning Scores (NEWS2) are more likely to die, require ICU admission, and have longer length of stay than patients with lower scores. 
- NEWS2 values are good predictors of short-term mortality in COVID-19 patients.

- Individually, the respiratory components (respiratory rate, oxygen saturation and supplemental oxygen requirement) are of the most value.

\section{Introduction}

The National Early Warning Score (NEWS) was developed in 2012 by the Royal College of Physicians (RCP) to detect deterioration and improve outcomes in hospital patients. ${ }^{1}$ NEWS comprises respiratory rate, oxygen saturation, temperature, systolic blood pressure (SBP), pulse, and level of consciousness. Each is scored 0-3 and combined to give an overall score with two additional points for supplemental oxygen. Scores lie from 0-20, with higher scores resulting from abnormal physiological measurements. The updated NEWS2 is similar, with the additional consideration of new onset confusion alongside level of consciousness and a new oxygen saturation scale (scale 2) for hypercapnic respiratory failure patients. ${ }^{2}$ NEWS2 is mandated by NHS England and NHS Improvement for use in acute hospital settings and the ambulance service, and is recommended for use in out-of-hospital settings including general practice and community care. . $^{1,3-6}$

COVID-19 is an infectious disease, typically characterised by a dry cough, shortness of breath, fever, fatigue, and/or loss of taste and smell. ${ }^{7}$ For many, symptoms are mild, but for some the disease is fatal. ${ }^{8,9}$ In March 2020, the World Health Organisation declared COVID-19 a pandemic. ${ }^{10}$

The RCP recommends that NEWS2 should be used when managing patients with COVID-1911; however, a recent review concluded that there is currently no evidence regarding the value of NEWS2 in COVID-19 patients outside hospital. ${ }^{12}$ In addition, remote GP consultations and the proposal for virtual wards makes recording NEWS2 difficult. There is therefore a need for evidence about whether NEWS2 and which component parts have value in identifying deterioration and poor outcomes in a COVID-19 population.

The aim of this study was to examine NEWS2 values and their component scores in hospitalised patients with COVID-19 in the West-of-England, and to investigate their relationship with hospital mortality and suitability as a prognostic tool.

\section{Methods}

\section{Design and setting}

This was a pragmatic, rapid, observational cohort study of COVID-19 positive patients admitted to North Bristol NHS Foundation Trust, University Hospitals Bristol and Weston NHS Foundation Trust, Great Western Hospitals NHS Foundation Trust, or Royal United Hospitals Bath NHS Foundation Trust, in the West-of-England. 
Patients were eligible if they were aged $\geq 16$ years at admission and had a positive swab taken during their hospital stay or up to 2-weeks before. Patients admitted to hospital more than once following a positive test are described, but only first admissions were included in analyses.

\section{Data acquisition}

Routinely collected electronic hospital data were requested from each of the four acute trusts. Data were anonymised prior to transfer to the research team. As data were routine and anonymised, patient consent was not required.

\section{Data}

Data were requested on eligible patients from the first case in each trust (early-March 2020) up until data extraction (approximately end of June 2020). As each trust extracted data at different times, data were amended to reflect the situation on June $27^{\text {th }} 2020$ (the date of first data extraction). All electronically recorded NEWS2 component physiological measurements (respiratory rate, oxygen requirement, oxygen saturation, hypercapnic respiratory failure status, systolic blood pressure, pulse, temperature, and level of consciousness [alert, voice, pain, unresponsive, newly confused]) were requested, along with date and time of each set of observations. Electronic observations were not recorded in intensive care units (ICU) in any of the four trusts, in the emergency departments (ED) except for Great Western, or the respiratory admission unit in Bath. Patient age, sex, hospital admission date, COVID-19 swab and result dates, ICU admission, hospital discharge status (hospital death, discharged, inpatient), and hospital discharge/death date were also requested.

\section{NEWS2 and component scores}

Each component score (e.g. temperature score of 0-3) and corresponding NEWS2 value was calculated from the physiological measurements listed above following the rules on the NEWS2 scoring card (Appendix 1).

'Baseline' was the day the positive COVID-19 swab was taken. All observations recorded before baseline were discarded. The 'first' NEWS2 value and component scores for each patient were derived from the first complete set of electronically recorded observations between baseline and 2 days post-baseline. Maximum NEWS2 values were the highest calculated NEWS2 values between baseline and discharge/death for each patient; maximum component scores were the highest component scores in this same period. NEWS2 change scores were calculated as maximum score minus first score.

NEWS2 values were grouped into four categories for analysis: $0-2,3-4,5-6$,and 7+, in line with hospital escalation trigger scores of 3,5 and $7 .{ }^{5}$

\section{Outcomes}


The primary outcome was mortality/ICU status: died in hospital and received ICU care, died without ICU admission, received ICU care prior to discharge, or discharged without ICU admission (patients who were inpatients when data were extracted are described but excluded from all analyses). Time to death for patients who died, and post-COVID-19 length of hospital stay (LOS) for patients who survived to discharge, were secondary outcomes. Time to death was calculated as date of death minus date of first score. LOS was calculated as discharge date minus COVID-19 swab date.

\section{Statistical analysis}

Continuous data were summarised using medians \& interquartile ranges (IQR). Categorical data were summarised using counts \& percentages.

The distribution of NEWS2 values and how this differed by mortality/ICU status were explored graphically. This analysis was split into three parts: first NEWS2 values, maximum NEWS2 values, and the change from first to maximum. Similarly, distributions of each component score were explored. These analyses were purely descriptive.

The sensitivity and specificity of first NEWS2 at different cut-offs were explored for 2-, 7- and 30-day mortality. Receiver Operating Characteristic (ROC) curves (sensitivity against 1-specificity) were constructed and area under the curve (AUC) calculated. As prespecified, AUC values of 0.7-0.79 were considered acceptable, 0.8-0.89 excellent and $\geq 0.9$ outstanding. ${ }^{13}$ Youden's index was used to identify the cut-off which best balanced the two measures. Sensitivity and specificity were used to predict the positive and negative predictive values (i.e. post-test probabilities) of NEWS2 at cut-offs of 3,5 and 7 to predict 2-day mortality, given different prevalence assumptions.

Stata 15.1 was used to conduct all data checking, cleaning, and analyses.

\section{Results}

\section{Demographics}

Between 11 March and 27 June 2020, 1,288 patients met the inclusion criteria. 541 (42\%) were female and median age was 74 years (IQR 59 to 84 ). 331 (26\%) died in hospital during their first admission, 40 of whom received ICU care before death, $83(6 \%)$ were admitted to ICU and subsequently discharged, and $849(66 \%)$ were discharged without ICU requirement. The remaining $25(2 \%)$ were inpatients on 27 Jun 2020 so were excluded from further analyses. Males were more likely to die than females ( $29 \%$ vs. $23 \%$ ), and older patients were more likely to die that younger patients $(2 \%, 18 \%, 34 \%$ and $45 \%$ of patients aged 16-49, 50-69, 70-89, and 90+ years, respectively).

Of those discharged, 196/932 (21\%) were readmitted (median time to first readmission 7 days, IQR 1 to 20); 147 were readmitted once, 31 twice, and 18 three-six times. 26 patients who were discharged following their first admission, died during a subsequent admission. All readmissions were excluded from 
analyses. 1,144 (91\%) patients had at least one set of complete electronic observations and 1,071 (85\%) had 'first' scores.

\section{NEWS2 values}

Most patients had low first NEWS2 values: 535 (50\%) NEWS2=0-2, 287 (27\%) NEWS2=3-4, 150 (14\%) NEWS2 $=5-6$, and $99(9 \%)$ NEWS2 $=7+$. However, many patients had a high score at some point during their stay: 156 (14\%) had a maximum score of NEWS2=0-2, 251 (22\%) NEWS2=3-4, 195 (17\%) NEWS2=5-6, and $542(47 \%)$ NEWS2=7+. Patients with higher first scores were more likely to die than patients with lower first scores (e.g. $19 \%$ with first NEWS2 $=0-2$ died compared to $49 \%$ with NEWS2 $=7+$; Figure 1 ); this relationship was more pronounced for maximum scores, with a clear distinction at NEWS $2=7+(4 \%$ with maximum NEWS2 $=0-2$ died compared to $47 \%$ with NEWS2 $=7+$; Figure 1 ). Change scores had a similar distribution and relationship with mortality status as first scores (Appendix 2). Patients with higher first NEWS2 were also likely to die earlier (Figure 2). For patients who survived to discharge, the higher the first and maximum NEWS2 value, the longer the LOS (Appendix 3). For example, 49\%, 46\%, 33\% and $18 \%$ of patients had LOS $<7$ days with first scores of $0-2,3-4,5-6$, and $7+$, respectively; this was $74 \%, 54 \%, 37 \%$ and $16 \%$ respectively for maximum scores.

\section{NEWS2 component scores}

The components of first NEWS2 which scored (i.e. had a value $>0$ ) most often were oxygen requirement (45\%), oxygen saturation (39\%) and pulse (29\%; Figure 4). Maximum oxygen saturation scored in $88 \%$ of patients; for most other components, the number of patients who scored at some point was between 67$76 \%$, except for consciousness which only ever scored in $16 \%$ of patients (Figure 3 ). In general, the higher the first or maximum component score, the higher the risk of hospital death (Figure 4). This relationship was less clear for first temperature and systolic blood pressure scores.

\section{Sensitivity, Specificity, AUC, PPV and NPV}

The AUC for 2-day, 7-day and 30-day mortality were $0.77,0.70$ and 0.65 , respectively. Figure 5 shows the sensitivity and specificity for 2-day mortality of NEWS2 cut-offs between 1 and 9 . According to Youden's Index (YI), 'best' cut-off was NEWS2=4 (YI=44.7); cut-offs of 3 ( $\mathrm{Yl}=39.3)$ and 5 ( $\mathrm{Yl}=36.7)$ may also be acceptable, depending on whether sensitivity or specificity was more important. The prevalence of 2-day mortality in this population was $3.8 \%$. Using the most common cut-offs of 3,5 , and 7 , post-test probabilities of 2-day mortality increased to $6.7 \%, 9.6 \%$ and $12.0 \%$ respectively for first scores $\geq$ the cutoff, and decreased to $0.9 \%, 2.1 \%$ and $3.0 \%$ respectively for patients with first scores below the cut-off (Table 1). Table 1 also details predicted values if we instead assume a prevalence of $1 \%$ or $0.5 \%$, which might better reflect patients seen in primary or community care. The AUCs for 2-day mortality for individual component scores were 0.71 for supplemental oxygen, 0.65 oxygen saturation, 0.64 respiratory rate, $0.56 \mathrm{SBP}, 0.53$ pulse, 0.53 temperature, and 0.53 consciousness. 
Table 1 Positive and negative predictive values (post-test probabilities) for 2-day mortality

\begin{tabular}{|lllll|}
\hline NEWS cut off & Prevalence & PPV & NPV & 1-NPV \\
\hline 3 & $3.8 \%$ & $6.7 \%$ & $99.1 \%$ & $0.9 \%$ \\
\hline 3 & $1 \%$ & $1.8 \%$ & $99.8 \%$ & $0.2 \%$ \\
\hline 3 & $0.5 \%$ & $0.9 \%$ & $99.9 \%$ & $0.1 \%$ \\
\hline 5 & & & & \\
\hline 5 & $3.8 \%$ & $9.6 \%$ & $97.9 \%$ & $2.1 \%$ \\
\hline 5 & $1 \%$ & $2.6 \%$ & $99.5 \%$ & $0.5 \%$ \\
\hline 7 & $0.5 \%$ & $1.3 \%$ & $99.7 \%$ & $0.3 \%$ \\
\hline 7 & & & & \\
\hline 7 & $3.8 \%$ & $12.0 \%$ & $97.0 \%$ & $3.0 \%$ \\
\hline
\end{tabular}

PPV=Positive predictive value. NPV=Negative predictive value.

\section{Discussion}

\section{Summary of Results}

This study included 1,288 COVID-19 positive hospitalised patients in the West-of-England. Patients were more likely to be male and older and these groups were also more likely to die, in line with other COVID-19 studies. ${ }^{9,14-16}$ Twenty-five percent died during their initial admission and a further $2 \%$ died during a subsequent admission. $10 \%$ of patients were admitted to ICU. The majority of first NEWS2 values were low ( $50 \%$ NEWS2=0-2, 27\% NEWS2=3-4), even though these patients were sick enough to be hospitalised; this is important when considering what threshold to use as an admission trigger in primary and community care. However, many patients went on to have high scores at some point during their hospital stay $(17 \%$ maximum NEWS2 $=5-6,47 \%$ maximum NEWS2 $=7+$ ). Patients with higher first NEWS2 values were more likely to require ICU admission and/or die, in line with findings from non-COVID-19, mostly prehospital, populations. ${ }^{17-19}$ Only $4 \%$ of patients with maximum NEWS2 $=0-2$ and $7 \%$ with maximum NEWS2 $=3-6$ required ICU or died, compared to $47 \%$ of patients with maximum NEWS2 $=7+$. LOS for survivors increased as first and maximum NEWS2 increased. The AUC for 2-day mortality was 0.77 , reducing to 0.70 and 0.65 for 7-day and 30-day mortality; these AUCs were considered acceptable at 2and 7-days according to our predefined criteria ${ }^{13}$, and support previous research suggesting early warning scores are best at predicting short-term outcomes. ${ }^{20,21}$ An increase in most first and maximum 
component scores was associated with an increased risk of hospital mortality, although this relationship was less clear for temperature and systolic blood pressure. For 2-day mortality, only oxygen requirement had an AUC which met the threshold for acceptable (0.71); oxygen saturation and respiratory rate were marginally predictive (AUC 0.65 and 0.64 , respectively), but the other components alone were not predictive at all (AUCs 0.53-0.56).

\section{Strengths and limitations}

The main strength of this paper is the inclusion of all patients with a positive COVID-19 diagnosis over a period of more than three months admitted to four hospitals encompassing communities of varying ages and deprivation. This enables our findings to be generalisable across the UK. A key limitation was the absence of electronic observations in ICU, three EDs, or the respiratory admission unit in Bath. This meant that $17 \%$ of patients were not included in the analysis of first scores, $10 \%$ were not included in maximum score analysis, and maximum scores may not have been true maximums. We would have liked to look at pre-hospital NEWS2, however without data linkage this would have meant relying on suspected COVID-19 status rather than confirmed. In addition, remote GP consultation meant that observations, and therefore NEWS2, in primary care data is often incomplete. We therefore focussed on hospital data for confirmed COVID-19 patients. A limitation of this approach was that $45 \%$ of patients scored for supplemental oxygen on first score which does not reflect the situation in primary care. However, it is likely that the score for supplemental oxygen would be replaced by a score for hypoxia in the community.

\section{Comparison with other literature}

NEWS2 in COVID-19 patients

There are a few small studies looking at NEWS2 in patients with COVID-19, but none in UK populations. A study of 66 patients in Norway ${ }^{22}$ admitted to ED found that NEWS2 predicted outcomes and performed better than other commonly used clinical risk scores qSOFA, SIRS and CRB-65. $42 \%$ of patients had NEWS $2 \geq 5$ at presentation compared to $23 \%$ with NEWS $\geq 5$ in our study. This is probably because many of our patients were already receiving treatment when their first score was recorded. A study of 68 admissions to an Italian hospital ${ }^{23}$ confirmed our finding that ICU admission was more likely for patients with higher NEWS2.

A group in China described how they added 3 points to NEWS for age $>65$ to predict severe COVID-19 disease, but did not report any outcome measures. ${ }^{24}$ Similarly, a UK group wrote a letter to the editor of BMJ stating that NEWS2 requires modification for COVID-19 patients, to account for oxygen demand rather than a binary component for oxygen delivery. ${ }^{25}$ The RCP have released revised guidance making staff aware that an increase in oxygen requirements should be a trigger for re-assessment. 
Hypoxia (low oxygen saturation) has been shown to predict COVID-19 mortality in other studies, for example, Xie et al found that higher Sp02 levels after oxygen supplementation were associated with reduced mortality, independent of age and sex. ${ }^{16,22,26}$ High respiratory rate has also been found to predict poor outcomes in this population ${ }^{27}$, as has low systolic blood pressure. ${ }^{28}$

Concerns have emerged regarding 'silent hypoxia', where a patient has low oxygen saturations but normal respiratory rate. ${ }^{29}$ We found 57/1,071 first scores had low oxygen saturation (scoring 3 for that NEWS2 component), and of those $28 / 57$ (49\%) had a normal respiratory rate (12-20 breath per minute); this was more common for patients without supplemental oxygen $(14 / 23,61 \%)$ that with oxygen $(14 / 34,41 \%)$. Therefore, silent hypoxia did exist in these patients, but numbers were small.

Pyrexia (high temperature) is one of the key COVID-19 symptoms described by the NHS ${ }^{30}$, but only $19 \%$ of our COVID-19 positive patients had a temperature $>38$ degrees at first score. Other authors ${ }^{9}$ found pyrexia to occur in only $30 \%$ of patients, but it has been reported in up to $79 \% .^{22}$

\section{Implications for research and/or practice}

An evidence review of NEWS2 and COVID-19 ${ }^{12}$ raised three research questions for the use of NEWS2 in primary care. The first was whether NEWS2 is valid as a measure of severity in COVID-19 and does it predict who is likely to deteriorate? We have demonstrated that NEWS2 predicts mortality, especially in the short term. The second question was whether a single NEWS2 score is sufficiently sensitive and specific? Again, we have shown that a single score can predict short-term mortality, and based on the AUC, NEWS2 $=4$ is the best value to balance sensitivity and specificity. A low score suggests that mortality is unlikely in the subsequent 2 days but over time scores deteriorate in many patients, so the use of serial scores is likely to be superior to a single score. In either case, NEWS2 should always be used alongside clinical judgement and not as a rule in/rule out test. The final question was whether NEWS is likely to be practical? Some components are measurable at home, but blood pressure and oxygen saturation require equipment. We found blood pressure alone was a poor predictor of mortality in the short term, but that oxygen saturations and respiratory rate are the most predictive, as supported by other studies. This suggests that the proposed role out of pulse oximeters for remote monitoring ${ }^{4,31}$ is likely to be valuable.

Further research measuring NEWS2 in COVID-19 patients in primary and community care is required. However, given that COVID-19 status may not be confirmed or recorded in the community, a retrospective study is likely to be difficult. NEWS2 is mandated in the UK acute sector, and used widely in primary and community care, so a randomised controlled trial is also unlikely to be possible. Linking ambulance data to hospital data would provide some of the picture, but obtaining linked data is often difficult and slow. Given these difficulties, we believe the evidence presented in this study can be applied in primary/community care settings, despite being collected in hospitalised patients. 
This study has demonstrated that NEWS2 is associated with mortality in COVID-19 patients. In particular, NEWS2 is a reasonably good predictor of 2-day mortality in this population, and the respiratory components (respiratory rate, oxygen saturation and supplemental oxygen requirement) are of the most value in the short term. These findings support the RCP's recommendations to use NEWS2, alongside clinical judgement, in the assessment of COVID-19 patients.

\section{Declarations}

\section{Acknowledgements}

We would like to thank Mike Wyatt, Kwok Lee, Jennifer Stonham and Tom Frost, from the business intelligence teams in each trust, for providing us with the data for this project and assisting with data queries.

\section{Funding statement:}

This research was jointly funded by the National Institute for Health Research (NIHR) Applied Research Collaboration West (ARC West) at University Hospitals Bristol and Weston NHS Foundation Trust (core NIHR infrastructure funded: NIHR200181) and the West of England Academic Health Science Network (core funded). The views expressed are those of the authors and not necessarily those of NHS England, NHS Improvement, the NIHR or the Department of Health and Social Care.

\section{Competing Interests}

None declared

\section{Ethics Approval}

This study was approved by the University of Bristol Faculty of Health Sciences Research Ethics Committee (ID 105666) and the Health Research Authority (IRAS ID 284987).

\section{Clinical Trial Registration}

Not applicable

\section{PPI statement}

An established public involvement panel met to discuss preliminary qualitative findings from previous work about NEWS from our research group. The key findings relevant to this study were that they saw the value in using NEWS, but there was some anxiety around balancing NEWS with clinicians' clinical judgement. We have mentioned the importance of interpreting NEWS2 alongside clinical judgement in our discussion. Unfortunately, due to time restraints, we were unable to reconvene the panel to discuss findings from this work. 


\section{References}

1. Royal College of Physicians. National Early Warning Score (NEWS): Standardising the assessment of acute-illness severity in the NHS. London RCP 2012.

2. Williams B. The National Early Warning Score 2 (NEWS2) in patients with hypercapnic respiratory failure. Clin Med (Lond). 2019;19(1):94-5.

3. National Institute for Health and Clinical Excellence. Sepsis: recognition, diagnosis and early management. London; 2017. Report No.: NG51.

4. NHS England, NHS Improvement. Pulse oximetry to detect early deterioration of patients with COVID19 in primary and community care settings. 2020.

5. Royal College of Physicians. National Early Warning Score (NEWS) 2: Standardising the assessment of acute-illness severity in the NHS. London: RCP; 2017.

6. The Royal College of Emergency Medicine. Position Statement: National Early Warning Score (NEWS) for Adult Patients attending Emergency Departments. 2016.

7. Centre for Disease Control and Prevention. Symptoms of Coronavirus. 2020.

8. Intensive care national audit \& research centre (ICNARC). ICNARC report on COVID-19 in critical care 31 July 2020. 2020.

9. Richardson S, Hirsch JS, Narasimhan M, et al. Presenting Characteristics, Comorbidities, and Outcomes Among 5700 Patients Hospitalized With COVID-19 in the New York City Area. JAMA. 2020.

10. WHO Director-General's opening remarks at the media briefing on COVID-19 - 11 March 2020 [press release]. 2020.

11. Royal College of Physicians. NEWS2 and deterioration in COID-19. RCP; 2020.

12. Greenhalgh T, Treadwell J, Burrow R, et al. NEWS (or NEWS2) score when assessing possible COVID19 patients in primary care? : Centre for Evidence-Based Medicine, Nuffield Department of Primary Care Health Sciences, University of Oxford; 2020.

13. Mandrekar JN. Receiver operating characteristic curve in diagnostic test assessment. J Thorac Oncol. 2010;5(9):1315-6.

14. European Centre for Disease Prevention and Control. Rapid Risk Assessment: Coronavirus disease 2019 (COVID-19) in the EU/EEA and the UK- ninth update. 2020 23/04/2020.

15. Wu Z, McGoogan JM. Characteristics of and Important Lessons From the Coronavirus Disease 2019 (COVID-19) Outbreak in China: Summary of a Report of 72314 Cases From the Chinese Center for Disease Control and Prevention. JAMA. 2020.

16. Xie J, Covassin N, Fan Z, et al. Association Between Hypoxemia and Mortality in Patients With COVID-19. Mayo Clin Proc. 2020;95(6):1138-47.

17. Martin-Rodriguez F, Lopez-Izquierdo R, Del Pozo Vegas C, et al. A Multicenter Observational Prospective Cohort Study of Association of the Prehospital National Early Warning Score 2 and Hospital Triage with Early Mortality. Emerg Med Int. 2019;2019:5147808. 
18. Scott LJ, Redmond NM, Tavare A, et al. Association between National Early Warning Scores in primary care and clinical outcomes: an observational study in UK primary and secondary care. $\mathrm{Br} \mathrm{J}$ Gen Pract. 2020;70(695):e374-e80.

19. Smith GB, Prytherch DR, Meredith P, et al. The ability of the National Early Warning Score (NEWS) to discriminate patients at risk of early cardiac arrest, unanticipated intensive care unit admission, and death. Resuscitation. 2013;84(4):465-70.

20. Alam N, Hobbelink EL, van Tienhoven AJ, et al. The impact of the use of the Early Warning Score (EWS) on patient outcomes: a systematic review. Resuscitation. 2014;85(5):587-94.

21. Patel R, Nugawela MD, Edwards HB, et al. Can early warning scores identify deteriorating patients in pre-hospital settings? A systematic review. Resuscitation. 2018;132:101-11.

22. Myrstad M, Ihle-Hansen $H$, Tveita AA, et al. National Early Warning Score 2 (NEWS2) on admission predicts severe disease and in-hospital mortality from Covid-19 - a prospective cohort study. Scand J Trauma Resusc Emerg Med. 2020;28(1):66.

23. Gidari A, De Socio GV, Sabbatini S, et al. Predictive value of National Early Warning Score 2 (NEWS2) for intensive care unit admission in patients with SARS-CoV-2 infection. Infect Dis (Lond). 2020;52(10):698-704.

24. Liao X, Wang B, Kang Y. Novel coronavirus infection during the 2019-2020 epidemic: preparing intensive care units-the experience in Sichuan Province, China. Intensive Care Med. 2020;46(2):35760.

25. Lim NT, Pan D, Barker J. NEWS2 system requires modification to identify deteriorating patients with COVID-19. Clin Med (Lond). 2020;20(4):e133-e4.

26. Petrilli $\mathrm{CM}$, Jones $\mathrm{SA}$, Yang J, et al. Factors associated with hospital admission and critical illness among 5279 people with coronavirus disease 2019 in New York City: prospective cohort study. BMJ. 2020;369:m1966.

27. Goyal P, Choi JJ, Pinheiro LC, et al. Clinical Characteristics of Covid-19 in New York City. N Engl J Med. 2020;382(24):2372-4.

28. Sze S, Pan D, Williams CML, et al. Letter to the Editor: Variability but not admission or trends in NEWS2 score predicts clinical outcome in elderly hospitalised patients with COVID-19. J Infect. 2020.

29. Healthcare Saefty Investigation Branch (HSIB). National Intelligence Report Early warning scores to detect deterioration in Covid-19 inpatients 2020 July 2020.

30. NHS. Check if you or your child has coronavirus symptoms 2020 [updated 14/08/2020. Available from: https://www.nhs.uk/conditions/coronavirus-covid-19/symptoms/.

31. Thornton J. The "virtual wards" supporting patients with covid-19 in the community. BMJ. 2020;369:m2119.

\section{Figures}




\section{First scores}



Maximum scores

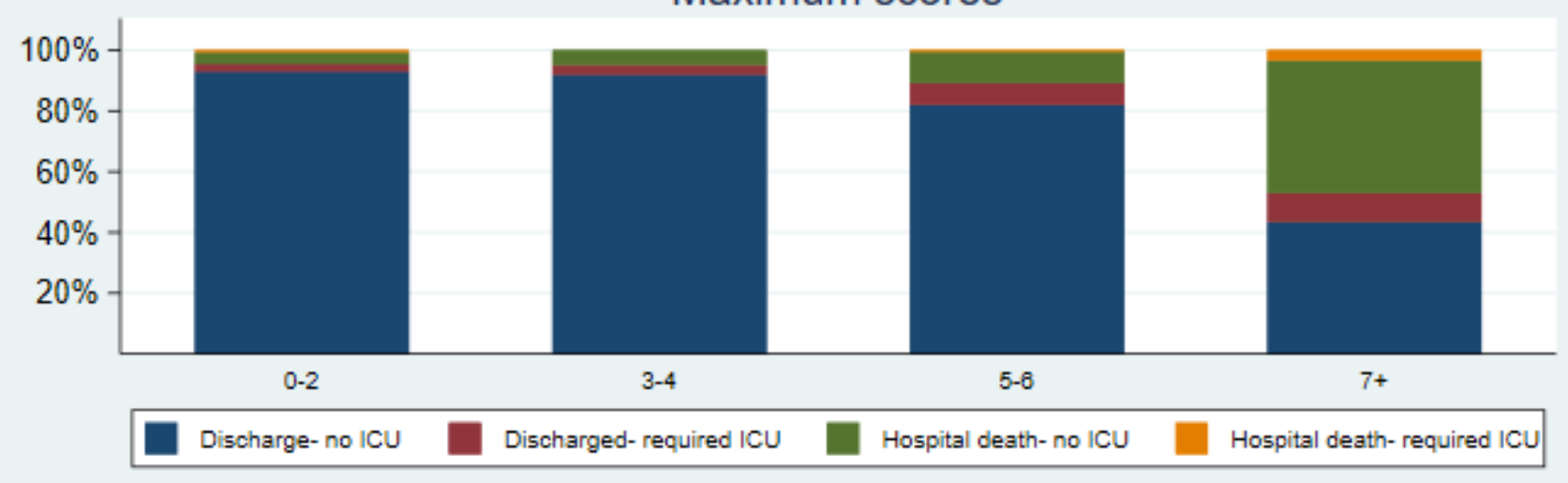

Figure 1

Mortality status by first and maximum NEWS2 


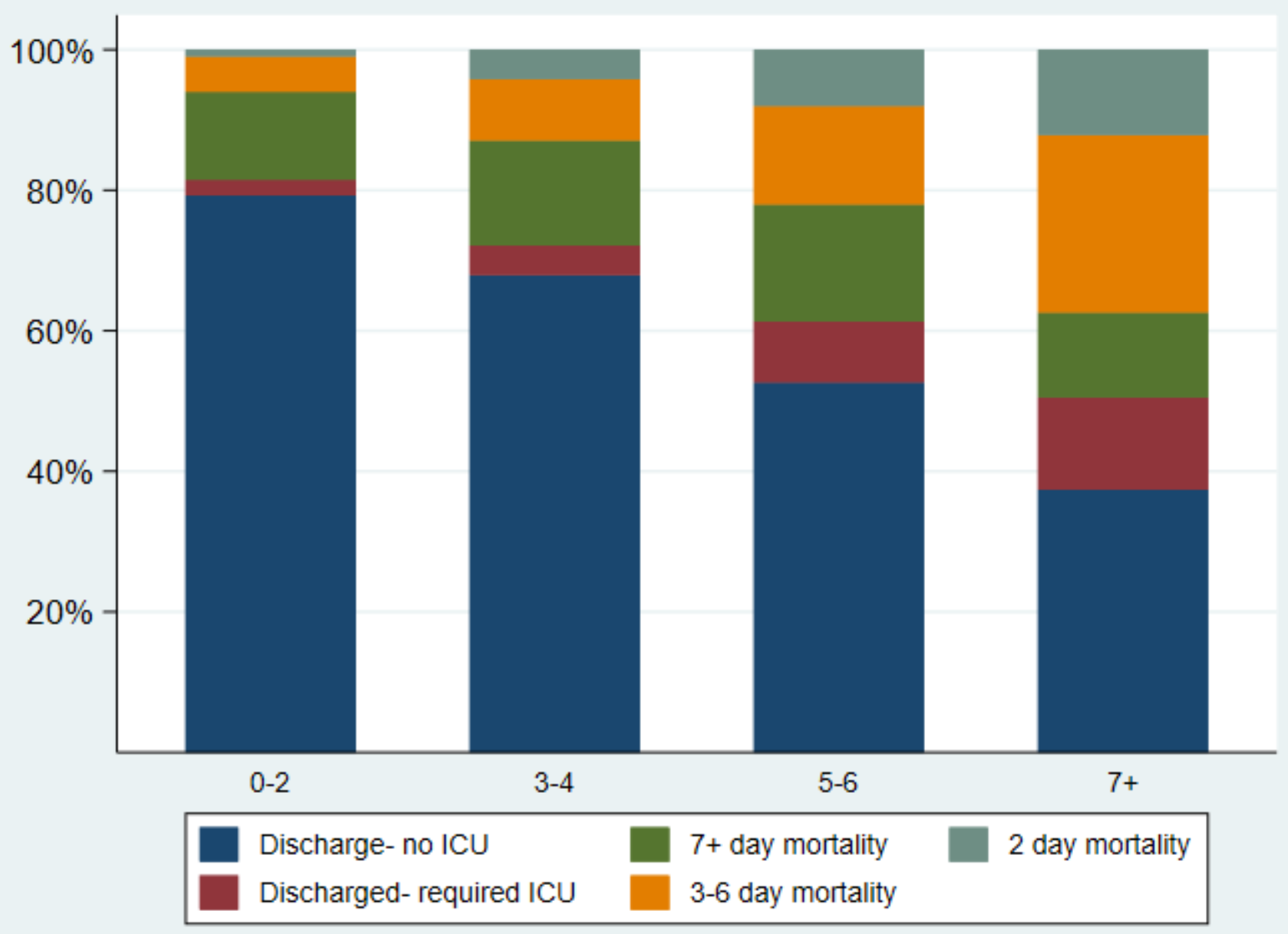

Figure 2

Mortality status and time to death by first NEWS2 


\section{First scores}

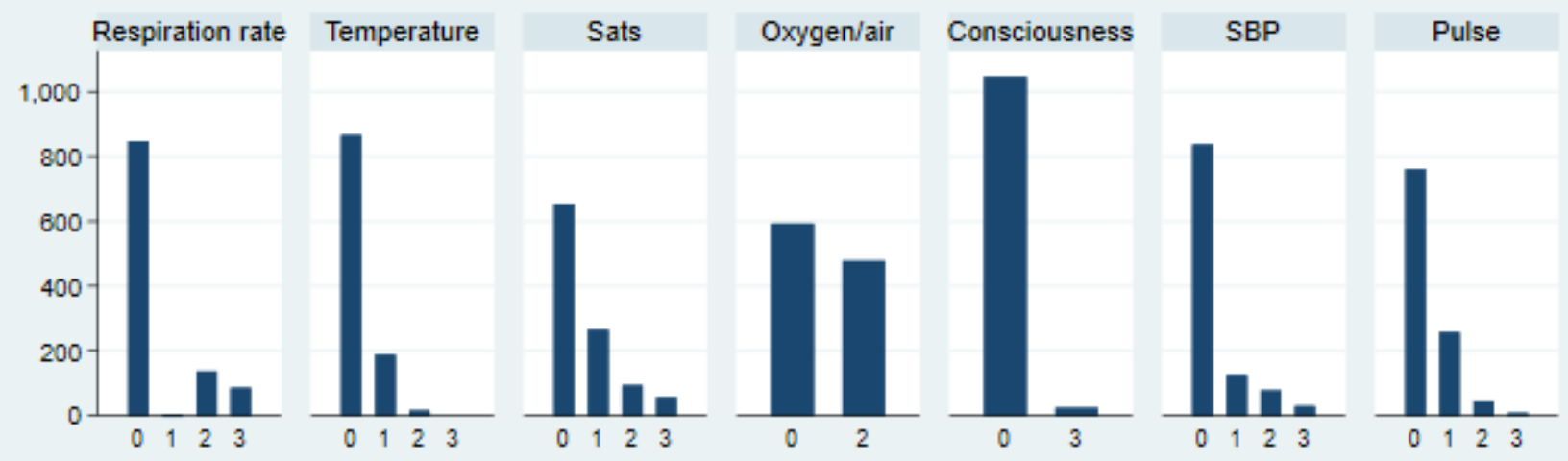

Maximum scores
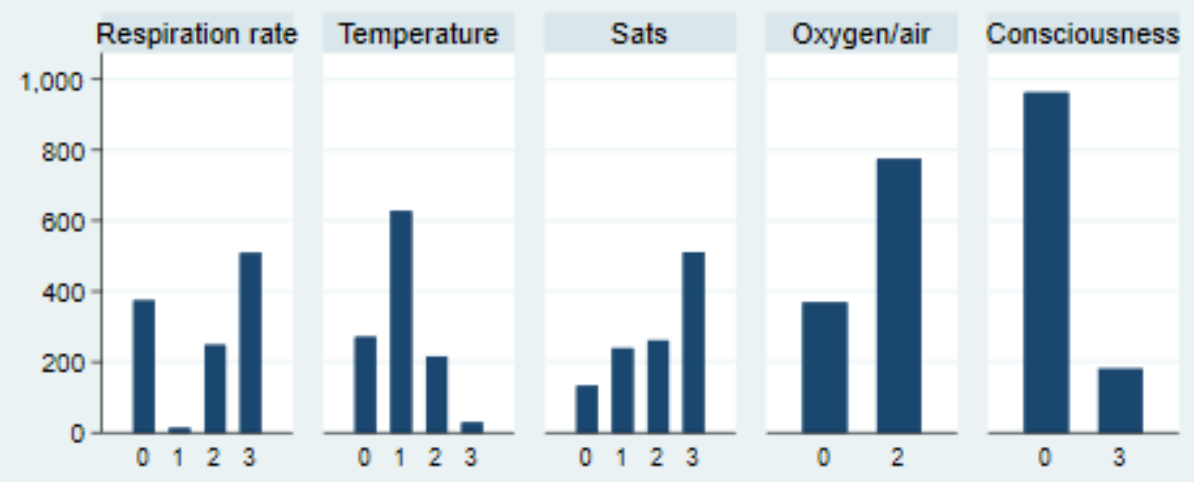

SBP

Pulse

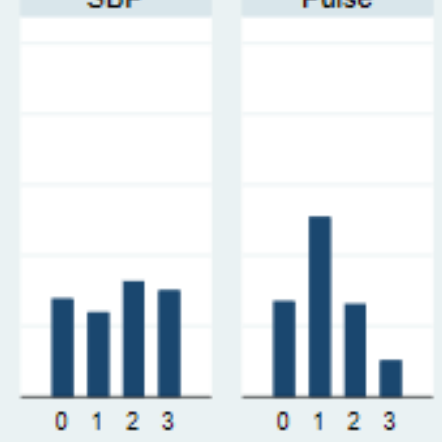

Figure 3

First and maximum component NEWS2 scores 
First scores


Pulse

Maximum scores


Pulse

\section{Figure 4}

Mortality status by first and maximum component NEWS2 scores 


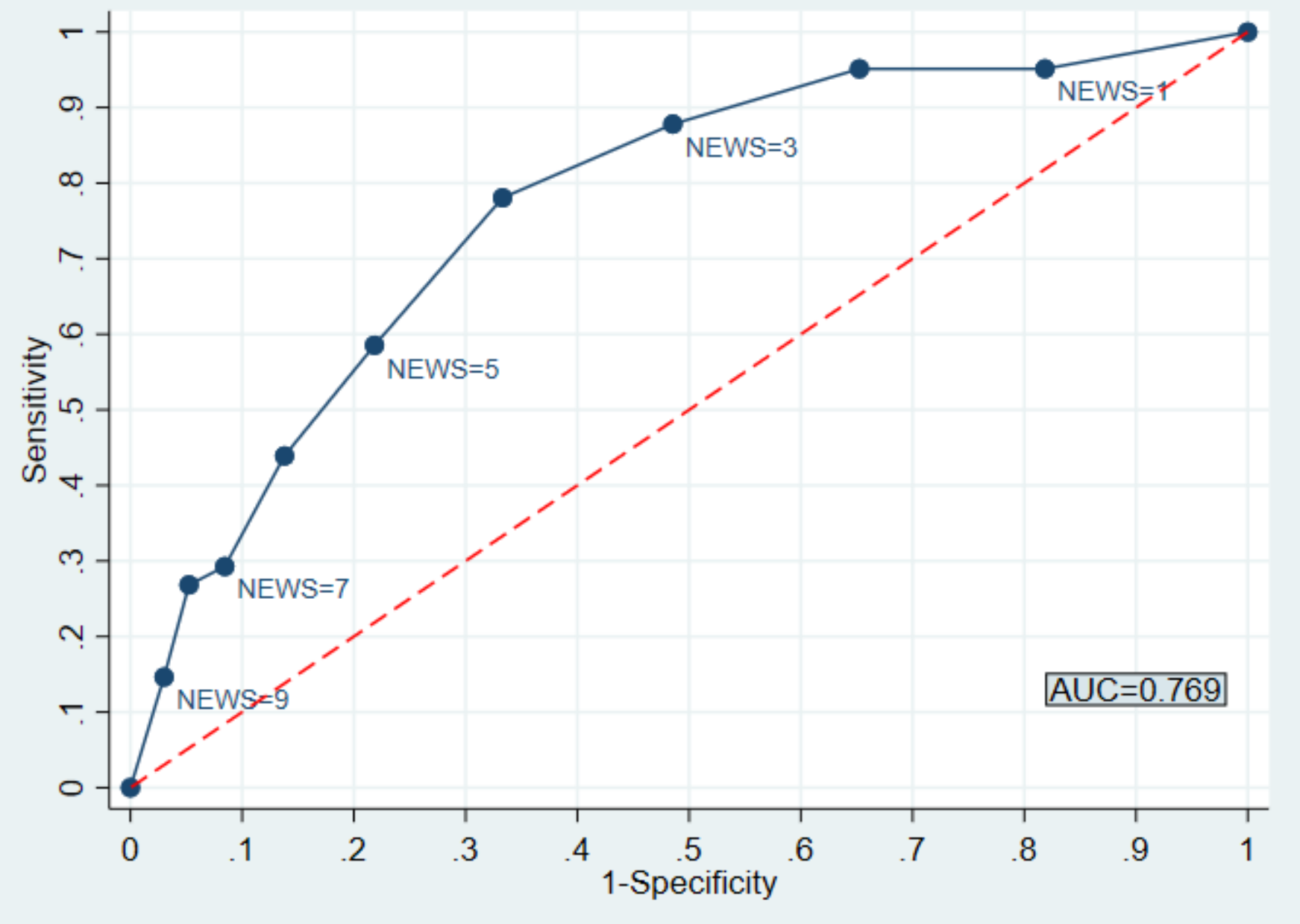

Figure 5

Receiver operating characteristic curve for 2-day mortality

\section{Supplementary Files}

This is a list of supplementary files associated with this preprint. Click to download.

- Supplementalfile.docx 